\title{
Preparando Pacientes e Otimizando Processos no Perioperatório das Cirurgias Cardíacas: Como Redesenhar os Fluxos de Assistência após a COVID-19
}

\author{
Preparing Patients And Optimizing Processes In The Perioperative Period Of Cardiac Surgery: How To \\ Redesign The Flow Of Care After COVID-19
}

Omar Asdrúbal Vilca Mejia, ${ }^{1,2}$ Bruno Mahler Mioto, ${ }^{\circledR}$ Gabrielle Barbosa Borgomoni, ${ }^{\circledR}$ Juliana Mendanha Camilo, ${ }^{\circledR}$ Danielle Misumi Watanabe, ${ }^{1}$ Sirlei Pereira Nunes, ${ }^{1}$ Vanessa Santos Sallai, ${ }^{1}$ Maraina Pegorini Libório de Lima, ${ }^{1}$ Jurema da Silva Herbas Palomo, ${ }^{1}$ Helenice Moreira da Costa, ${ }^{1}$ Elisandra Trevisan Arita, ${ }^{1}$ Maria Ignêz Zanetti Feltrim, ${ }^{1}$ Vera Coimbra, ${ }^{1}$ Roger Daglius Dias, ${ }^{3}$ Filomena Regina Barbosa Gomes Galas, ${ }^{1}$ José Otávio Costa Auler Junior, ${ }^{1}$ Fabio Biscegli Jatene Instituto do Coracao (InCor), Hospital das Clinicas HCFMUSP, Faculdade de Medicina, Universidade de Sao Paulo, ${ }^{1}$ Sao Paulo, SP - Brasil Hospital Samaritano Paulista, ${ }^{2}$ São Paulo, SP - Brasil

Harvard Medical School, ${ }^{3}$ Boston, Massachusetts - EUA

\section{Introdução}

A falta de estratégias proativas de cuidados influenciam a fragmentação dos cuidados, desparametrização de processos e prolongamento de tempos hospitalares, ${ }^{1}$ enquanto evidências mostraram que protocolos multiprofissionais baseados em evidências executados por equipes sincronizadas, com padronização de processos, proatividade e cuidados centrados no paciente diminuem complicações, tempos e custos hospitalares. ${ }^{2,3}$ Neste contexto, cuidados baseados no conceito Enhanced Recovery After Surgery (ERAS) revolucionam os fluxos tradicionais. ${ }^{4}$ Diretrizes para cirurgia cardíaca foram publicadas recentemente, ${ }^{5}$ alcançando resultados encorajadores. ${ }^{6,7}$

Esta abordagem apontou eficiência e segurança na alta hospitalar em três dias após cirurgia cardíaca, ${ }^{6,8}$ algo promissor na era COVID-19, em que as filas cirúrgicas cresceram por adiamentos procedimentais, possibilitando maior número de atendimentos em menor tempo, redução do risco de contaminação e custos hospitalares. ${ }^{9}$

O Instituto do Coração, um dos maiores centros de cirurgia cardíaca,10 produziu um manual multiprofissional de cuidados baseado no conceito ERAS, otimizando processos através do preparo de pacientes para rápida recuperação após cirurgia cardíaca. A figura 1 apresenta os objetivos da implementação do fluxo Tempos Certos e seu possível impacto.

\section{Palavras-chave}

Melhoria de Qualidade; Segurança do Paciente; Procedimentos Cirúrgicos Cardiovasculares; COVID-19; Recuperação Pós-Cirúrgica Melhorada.

Correspondência: Omar Asdrúbal Vilca Mejia •

Instituto do Coração (InCor), Hospital das Clínicas HCFMUSP, Faculdade de Medicina, Universidade de São Paulo - Av. Dr, Enéas de Carvalho Aguiar, 44 CEP 05403-900, São Paulo, SP - Brasil

E-mail: omar.mejia@incor.usp.br

Artigo recebido em 03/06/2021; revisado recebido em 28/07/2021; aceito em 28/07/2021

DOI: https://doi.org/10.36660/abc.20210484

\section{Parecer}

O manual Tempos Certos foi elaborado por representantes multiprofissionais do Instituto do Coração do Hospital das Clínicas da Faculdade de Medicina da Universidade de São Paulo, baseando-se em evidências no preparo de pacientes para um rápido retorno às atividades após cirurgia. $\mathrm{O}$ conceito é dinâmico, por isso o documento será revisado periodicamente.

\section{Critérios de Inclusão}

Todo paciente programado para cirurgia cardíaca pode ser beneficiado pelos cuidados otimizados, quando coordenados por uma equipe multidisciplinar.

\section{Linha de Cuidados Tempos Certos}

A linha de cuidados transdisciplinar se inicia no ambulatório e é finalizada no seguimento após a alta hospitalar de cada paciente (Figuras 2 e 3).

\section{Ambulatório}

1. Avaliação da adequação da indicação.

2. Avaliação odontológica, psicológica (questionário SF36 — qualidade de vida — e HADS — escala hospitalar de ansiedade e depressão), fisioterapêutica (medidas de repouso, teste de caminhada de seis minutos, ventilometria, Short Physical Performance Battery e reabilitação pré-operatória), nutricional (orientações sobre abreviação de jejum), anestésica, de enfermagem e serviço social.

3. Coleta de vigilância: COVID-19.

\section{Pré-internação}

1. Programar e confirmar a internação segundo orientações multiprofissionais.

\section{Internação hospitalar}

1. Pacientes eletivos: admissão 6 horas antes do procedimento. 


\section{Carta Científica}

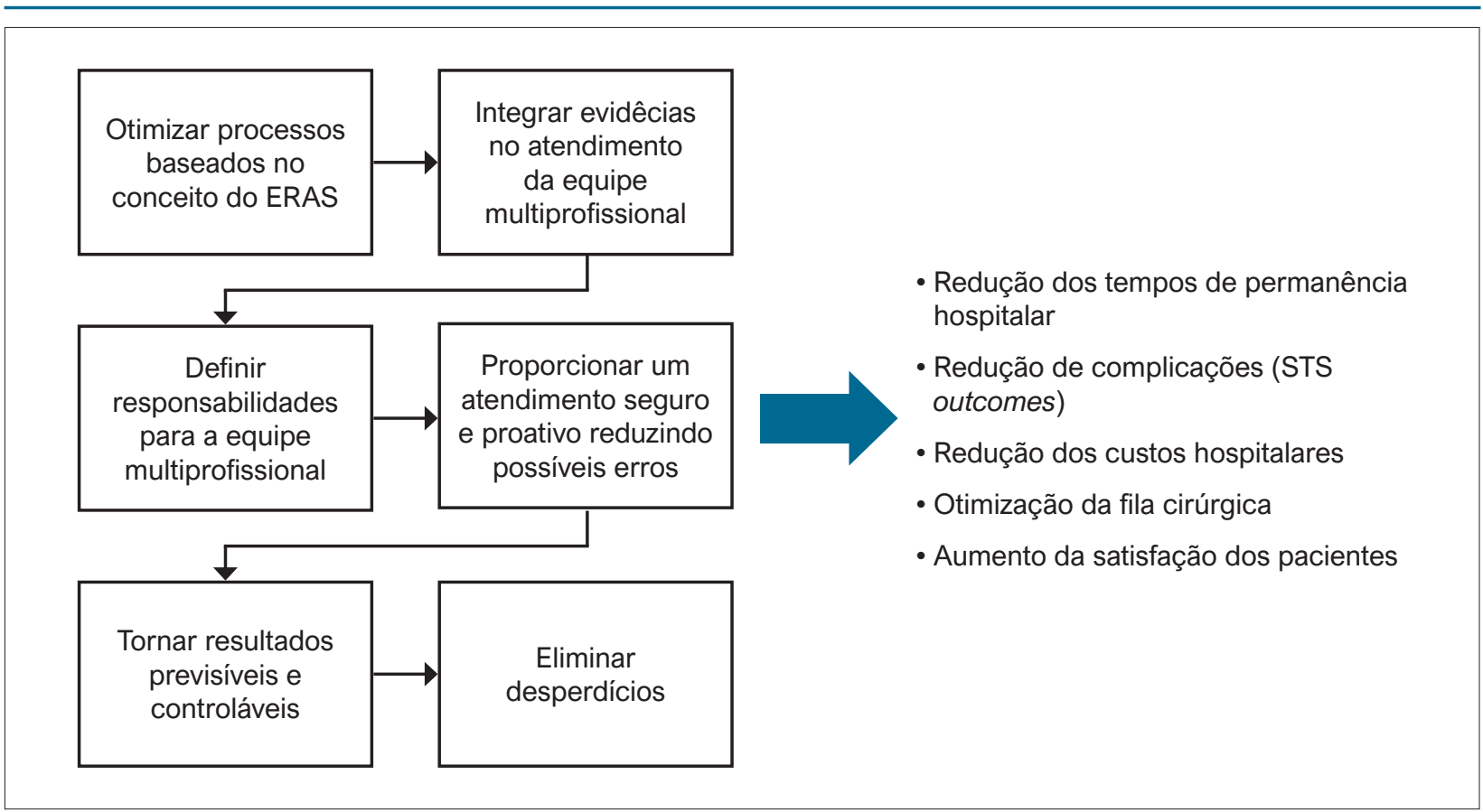

Figura 1 - Objetivos da linha de cuidados Tempos Certos. ERAS: Enhanced Recovery After Surgery.

\section{InCor: Tempos Certos}

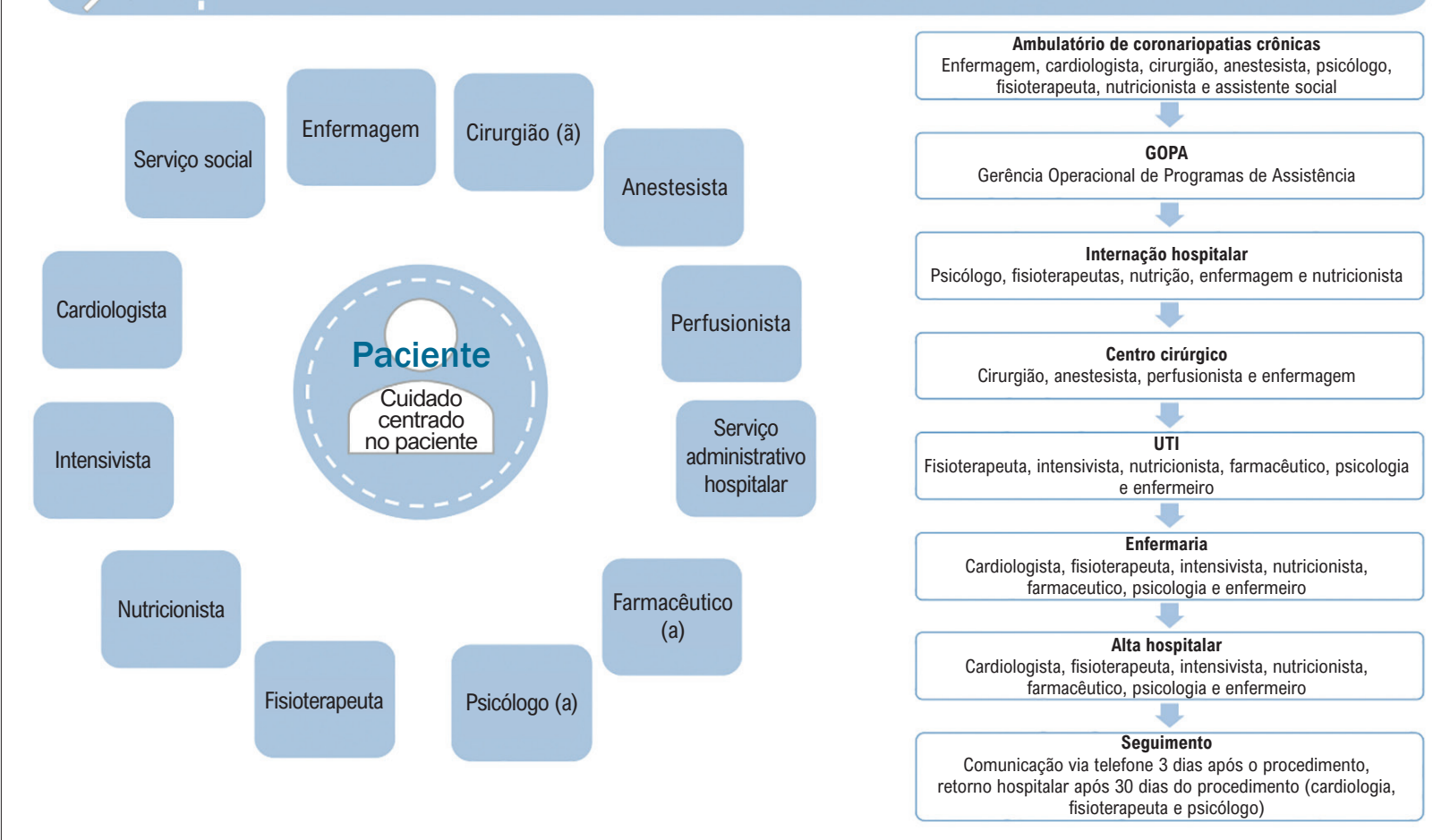

Figura 2 - Equipes envolvidas no fluxo Tempos Certos. 


\section{Tempos Certos: linha de cuidados}
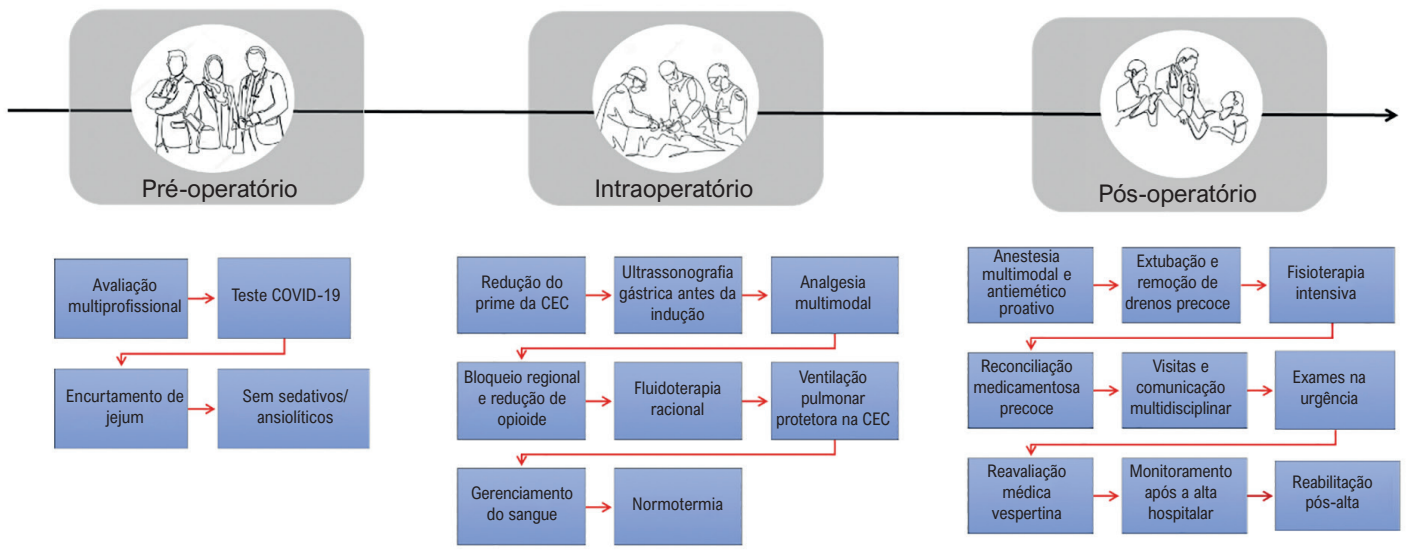

Figura 3 - Mapa dos processos perioperatórios: Tempos Certos. CEC: circulação extracorpórea.

2. Checagem da adesão ao protocolo no preparo prévio.

3. Refeição completa até 8 horas antes da indução anestésica.

4. Quebra do jejum 2 horas antes da cirurgia (líquido claro com maltodextrina, volume máximo $400 \mathrm{~mL}$ ).

5. Não prescrever sedativos e/ou ansiolíticos.

\section{Cirurgia}

1. Identificação paciente Tempos Certos.

2. Redução do circuito da circulação extracorpórea (CEC) e da hemodiluição (perfusato $<1000 \mathrm{~mL}$ ).

3. Ultrassonografia gástrica pré-indução anestésica.

4. Analgesia multimodal (podendo ser usados: sulfato de magnésio, lidocaína, dextrocetamina e dexametasona antes da incisão, e dipirona ao final da cirurgia).

5. Sedação e bloqueio regional (eretor da espinha).

6. Redução do uso de opioides, podendo fazer uso de rocurônio/cisatracúrio, ketamina, dexdetomidina, propofol, isofluorano ou sevofluorane e antiemético.

7. Fluidoterapia guiada por metas (alvo balanço zero).

8. Índice bispectral e monitorização de bloqueio neuromuscular train-of-four.

9. Ventilação pulmonar 3-5 mL/Kg durante a CEC.

10. Drenagem torácica anterior.

11. Normotermia.

12. Glicemia $<160 \mathrm{mg} / \mathrm{dL}$.

13. Gerenciamento do sangue.

14. Transporte do paciente intubado para a unidade de terapia intensiva (UTI) sob efeito residual da anestesia, portando OXILOG e bomba de infusão com propofol ou precedex.

\section{UTI}

1. Identificação paciente Tempos Certos.

2. Anestesia multimodal (ketamina em PCA (patientcontrolled analgesia), dipirona, dexametasona e tramal).

3. Antiemético preventivo.

4. Extubação em até 6 horas.

5. Pressão positiva contínua em vias aéreas (continuous positive airway pressure ou CPAP) por até 1 hora.

6. Reintrodução de ingestão oral (dieta líquida) quando houver lucidez (a partir de 2 horas após extubação).

7. Remoção dos drenos após redução da curva de sangramento (ultrassom confirma ausência de derrame).

8. Fisioterapia 6/6 horas: ausculta pulmonar e SpO2, estímulo à sedestação, exercícios respiratórios e motores, deambulação precoce, CPAP por 40 minutos.

9. Avaliação de enfermagem, incluindo avaliação delirium e dor: 1/1h até 12 horas de internação e $2 / 2$ horas quando $>12$ horas.

\section{Enfermaria}

1. Identificação paciente Tempos Certos.

2. Reconciliação medicamentosa precoce.

3. Equipe multidisciplinar intensifica visitas e comunicação.

4. Fisioterapia 6/6 horas: ausculta pulmonar e SpO2, estímulo à sedestação, exercícios respiratórios e motores, deambulação precoce, CPAP por 40 minutos. Dia da alta: realizar medidas de repouso, teste da caminhada de seis minutos (meta $>80 \%$ ), ventilometria e Short Physical Performance Battery (SPPB).

5. Reavaliação médica no período da tarde (exames status urgência).

6. Reavaliação psicológica e reaplicação de questionários. 


\section{Carta Científica}

7. Aconselhamento educacional, nutricional e psicológico para alta hospitalar.

\section{Seguimento (telessaúde ou presencial)}

1. Monitoramento após 3 dias da alta hospitalar.

2. Reabilitação fisioterápica (sinais vitais, SPPB, teste de caminhada 6 minutos e capacidade vital pulmonar).

3. Reavaliação psicológica e aplicação questionários. Em caso de demanda emocional, reabilitação (psicoterapia breve focal).

\section{Possíveis contraindicações}

Extubação precoce: sangramento importante, instabilidade hemodinâmica e respiratória e/ou falta de drive central respiratório.

Mobilização precoce: baixo débito cardíaco em uso de marcapasso epicárdico, instabilidade hemodinâmica (SVO2 <60, lactato alterado, noradrenalina 0,2 mcg/ $\mathrm{kg} / \mathrm{min})$, delirium, sangramento $>400 \mathrm{~mL}$ em $1 \mathrm{~h}>100$ $\mathrm{mL} / \mathrm{h}$ por $4 \mathrm{~h}$ seguidas, instabilidade respiratória - esforço respiratório.

\section{Considerações}

Os sistemas de saúde têm avançando pouco frente a indústrias de alto desempenho. A chegada da COVID-19 impõe mudanças aceleradas para lidar com a nova realidade. A implementação dos conceitos de rápida recuperação, que já apresentavam resultados positivos na era pré-pandemia, inclusive em nosso cenário, ${ }^{6}$ mais do que nunca se tornaram necessárias para lidar com a demanda reprimida e ao mesmo tempo reduzir a exposição desnecessária do paciente ao ambiente hospitalar. O trabalho em equipe multidisciplinar, de forma sincronizada e harmônica, conseguiria adotar uma abordagem centrada no paciente, otimizando processos, melhorando a assistência e a segurança do paciente,

\section{Referências}

1. Clay-Williams R, Taylor N, Ting HP, Winata T, Arnolda G, Austin E, et al. The Relationships Between Quality Management Systems, Safety Culture and Leadership and Patient Outcomes in Australian Emergency Departments. Int J Qual Health Care. 2020;32(Suppl 1):43-51. doi: 10.1093/intghc/mzz105.

2. Lee L, Mata J, Ghitulescu GA, Boutros M, Charlebois P, Stein B, et al. Cost-Effectiveness of Enhanced Recovery Versus Conventional Perioperative Management for Colorectal Surgery. Ann Surg. 2015;262(6):1026-33. doi: 10.1097/SLA.0000000000001019.

3. Gustafsson UO, Hausel J, Thorell A, Ljungqvist O, Soop M, Nygren J. Adherence to the Enhanced Recovery After Surgery Protocol and Outcomes After Colorectal Cancer Surgery. Arch Surg. 2011;146(5):571-7. doi: 10.1001/archsurg.2010.309.

4. Ljungqvist $\mathrm{O}$, Scott $\mathrm{M}$, Fearon KC. Enhanced Recovery After Surgery: A Review. JAMA Surg. 2017;152(3):292-8. doi: 10.1001/ jamasurg.2016.4952. assim como ampliando o acesso à saúde. Desta forma, conseguiríamos gerar valor no atendimento dos pacientes para a sustentabilidade dos programas de cirurgia cardíaca.

\section{Contribuição dos autores}

Concepção e desenho da pesquisa: Mejia OAV, Borgomoni GB, Jatene FB; Redação do manuscrito: Mejia OAV, Mioto BM, Borgomoni GB, Camilo JM, Watanabe DM, Nunes SP, Sallai VS, Lima MPL, Palomo JSH, Costa HM, Arita ET, Feltrim MIZ, Coimbra V; Revisão crítica do manuscrito quanto ao conteúdo intelectual importante: Mejia OAV, Mioto BM, Borgomoni GB, Camilo JM, Watanabe DM, Nunes SP, Sallai VS, Lima MPL, Palomo JSH, Costa HM, Arita ET, Feltrim MIZ, Coimbra V, Dias RD, Galas FRBG, Auler Junior JOC, Jatene FB.

\section{Potencial conflito de interesse}

Não há conflito com o presente artigo

\section{Fontes de financiamento}

O presente estudo não teve fontes de financiamento externas.

\section{Vinculação acadêmica}

Este artigo é parte de Estudo prospectivo e randomizado para avaliação do impacto do fluxo de rápida recuperação no tempo de pós-operatório em cirurgia de revascularização miocárdica de Gabrielle Barbosa Borgomoni e Omar Asdrúbal Vilca Mejia pela Universidade de São Paulo - Faculdade de Medicina.

\section{Aprovação ética e consentimento informado}

Este artigo não contém estudos com humanos ou animais realizados por nenhum dos autores.

5. Engelman DT, Ben Ali W, Williams JB, Perrault LP, Reddy VS, Arora RC, et al. Guidelines for Perioperative Care in Cardiac Surgery: Enhanced Recovery After Surgery Society Recommendations. JAMA Surg. 2019;154(8):755-66. doi: 10.1001/jamasurg.2019.

6. Mejia OAV, Borgomoni GB, Lasta N, Okada MY, Gomes MSB, Foz MLNN, et al. Safe and Effective Protocol for Discharge 3 Days After Cardiac Surgery. Sci Rep. 2021;11(1):8979. doi: 10.1038/s41598-02188582-0.

7. Grant MC, Isada T, Ruzankin P, Whitman G, Lawton JS, Dodd-O J, et al. Results from an Enhanced Recovery Program for Cardiac Surgery. J Thorac Cardiovasc Surg. 2020;159(4):1393-1402. doi: 10.1016/j. jtcvs.2019.05.035.

8. Maxwell YL. Safer, Quicker Discharge with Protocols for Enhanced Recovery After Surgery [Internet]. New York: Cardiovascular Research Foundation; 2020 [cited 2021 Jul 23]. p. 1-5. Available from: https:// www.tctmd.com/news/safer-quicker-discharge-protocols-enhancedrecovery-after-surgery. 
9. Gregory AJ, Grant MC, Boyle E, Arora RC, Williams JB, Salenger R, et al. Cardiac Surgery-Enhanced Recovery Programs Modified for COVID-19: Key Steps to Preserve Resources, Manage Caseload Backlog, and Improve Patient Outcomes. J Cardiothorac Vasc Anesth. 2020;34(12):3218-24. doi: 10.1053/j.jvca.2020.08.007.
10. Newsweek. World's Best Specialized Hospitals 2021 [Internet]. New York: Newsweek; 2021 [cited 2021 Oct 20]. p. 1-15. Available from: https://www.newsweek.com/worlds-bestspecialized-hospitals-2021?fbclid=IwAR1DrlrSj1-sBqwV0g4hyBjV2rLGGP4OnbMr3uB9L_9scz7ECv-TkOZ738. 